\title{
The Bohr compactification, modulo a metrizable subgroup
}

\author{
by
}

W. W. Com fort (Middletown, Conn.),

F. Javier Trigos-A r riet a (Bakersfield, Calif.), and Ta-Sun Wu (Cleveland, Ohio)

\begin{abstract}
The authors prove the following result, which generalizes a well-known theorem of I. Glicksberg [G]: If $G$ is a locally compact Abelian group with Bohr compactification bG, and if $N$ is a closed metrizable subgroup of bG, then every $A \subseteq G$ satisfies: $A \cdot(N \cap G)$ is compact in $G$ if and only if $\{a N: a \in A\}$ is compact in bG/N. Examples are given to show: (a) the asserted equivalence can fail in the absence of the metrizability hypothesis, even when $N \cap G=\{1\}$; (b) the asserted equivalence can hold for suitable $G$ and $N$ with $N$ closed in bG but not metrizable; (c) an Abelian group may admit two topological group topologies $\mathcal{U}$ and $\mathcal{T}$, with $\mathcal{U}$ totally bounded, $\mathcal{T}$ locally compact, $\mathcal{U} \subseteq \mathcal{T}$, with $\mathcal{U}$ and $\mathcal{T}$ sharing the same compact sets, and such that nevertheless $\mathcal{U}$ is not the topology inherited from the Bohr compactification of $\langle G, \mathcal{T}\rangle$.

There are applications to topological groups of the form $\mathrm{k} G$ for $G$ a totally bounded
\end{abstract} Abelian group.

\section{Introduction: notation, definitions, and results from the literature}

1.1. The classes $\mathbf{T B A G}, \mathbf{M A P}$, and $\mathbf{L C A G}$. A topological group $G$ is said to be totally bounded if there is a compact group $K$ such that $G$ is a topological subgroup of $K$. When this occurs one may assume, replacing $K$ by $\mathrm{cl}_{K} G$ if necessary, that $G$ is dense in $K$. It is a theorem of A. Weil [W] that when $G$ is totally bounded the compact group $K$ containing $G$ densely is unique up to a topological isomorphism leaving $G$ fixed pointwise. We write $K=\bar{G}$ and we refer to $K$ as the Weil completion of $G$. We denote by TBAG the class of totally bounded Hausdorff (Abelian) topological groups.

Given an Abelian topological group $G=\langle G, \mathcal{T}\rangle$, we denote by $\widehat{G}$ or by $\langle G, \mathcal{T}\rangle^{\wedge}$ the set of continuous homomorphisms from $G$ into the circle

1991 Mathematics Subject Classification: 54H11, $22 \mathrm{~A} 05$.

Key words and phrases: Bohr compactification, totally bounded Abelian group, locally compact Abelian group, respects compactness, k-space, Weil completion. 
group $\mathbb{T}$. Writing $K=\mathbb{T}^{\hat{G}}$, we define $e: G \rightarrow K$ by

$$
(e x)_{h}=h(x) \quad \text { for } x \in G, h \in \widehat{G},
$$

and we set $\mathrm{b} G=\mathrm{cl}_{K} e[G]$; the group $\mathrm{b} G$ is the so-called Bohr compactification of $G$.

Clearly the homomorphism $e$ is an isomorphism if and only if $\widehat{G}$ separates points of $G$. The groups which satisfy this condition are said to be maximally almost periodic in the sense of von Neumann. We denote by MAP the class of maximally almost periodic (Abelian) groups. For $G=\langle G, \mathcal{T}\rangle \in \mathbf{M A P}$ we denote by $\mathcal{T}^{+}$the topology of $\mathrm{b} G$ (or, with minor abuse of notation, any one of its subspaces). In general we suppress explicit mention of the isomorphism $e$; we treat $G$ as a subset of bG. Thus $\left\langle G, \mathcal{T}^{+}\right\rangle$(alternative notation: $G^{+}$) denotes $G$ with the totally bounded topological group topology inherited from $\mathrm{b} G$; that is, $G^{+}$is $G$ with the weak topology induced by $\widehat{G}$. More generally, given $\langle G, \mathcal{T}\rangle \in \mathbf{M A P}$ and $A \subseteq G$, we denote by $\langle A, \mathcal{T}\rangle$ the set $A$ with the topology inherited from $G$ and by $\left\langle A, \mathcal{T}^{+}\right\rangle$the set $A$ with the topology inherited from $G^{+}$(that is, from bG).

For $\mathbf{P}$ a topological property and $G=\langle G, \mathcal{T}\rangle \in \mathbf{M A P}$ we say, following [T1], [T2], [CT], [T3], that $G$ respects $\mathbf{P}$ if every $A \subseteq G$ satisfies: $\langle A, \mathcal{T}\rangle \in \mathbf{P}$ if and only if $\left\langle A, \mathcal{T}^{+}\right\rangle \in \mathbf{P}$.

We denote by LCAG the class of locally compact, Abelian, Hausdorff topological groups. The class-theoretic inclusion LCAG $\subseteq$ MAP is crucial to the success of Pontryagin duality theory; see [HR](22.17) for a proof.

We say as usual, as in 3.1 below, that a (not necessarily locally compact) group $G$ satisfies Pontryagin duality if the evaluation function from $G$ into $\widehat{\widehat{G}}$ is a surjective homeomorphism and an isomorphism. It is evident that every group $G$ satisfying Pontryagin duality must satisfy $G \in$ MAP.

It is a well-known result of Glicksberg $[\mathrm{G}]$ that every $G \in$ LCAG respects compactness. (Throughout this paper, we refer to this statement as "Glicksberg's theorem.") There is a useful addendum to Glicksberg's theorem, noted by Glicksberg himself: If $A \subseteq G=\langle G, \mathcal{T}\rangle \in \mathbf{L C A G}$, then not only is $\langle A, \mathcal{T}\rangle$ compact if and only if $\left\langle A, \mathcal{T}^{+}\right\rangle$is compact but also (if these conditions hold) the identity function $i:\langle A, \mathcal{T}\rangle \rightarrow\left\langle A, \mathcal{T}^{+}\right\rangle$is a homeomorphism; this follows from the inclusion $\mathcal{T}^{+} \subseteq \mathcal{T}$ and the fact that a compact Hausdorff space admits no strictly finer Hausdorff topology.

It is shown in [T1], [T2], generalizing Glicksberg's theorem, that every $G \in \mathbf{L C A G}$ respects in addition the properties $\mathbf{P}=\sigma$-compact, $\mathbf{P}=$ Lindelöf, $\mathbf{P}=$ pseudocompact, and $\mathbf{P}=$ functionally bounded. For the second two of these properties $\mathbf{P}$ the identity function $e:\langle A, \mathcal{T}\rangle \rightarrow\left\langle A, \mathcal{T}^{+}\right\rangle$is again a homeomorphism if $\langle A, \mathcal{T}\rangle \in \mathbf{P}$, but for $\mathbf{P}=\sigma$-compact or $\mathbf{P}=$ Lindelöf the corresponding statement fails. (It is easy to see, for example, denoting by $\mathcal{T}$ the usual locally compact topology on the real line $\mathbb{R}$, that the containment 
$\mathcal{T}^{+} \subseteq \mathcal{T}$ is strict. Indeed, $\mathcal{T}^{+}$is a totally bounded group topology for $\mathbb{R}$, while $\mathcal{T}$ is not.)

1.2. Discussion. The fact that $\left\langle G, \mathcal{T}^{+}\right\rangle$is totally bounded whenever $\langle G, \mathcal{T}\rangle \in \mathbf{M A P}$ (in particular, whenever $\langle G, \mathcal{T}\rangle \in \mathbf{L C A G}$ ) suggests two natural questions.

(1) Let $\langle G, \mathcal{U}\rangle \in$ TBAG. Must there exist a topological group topology $\mathcal{T}$ for $G$ such that $\langle G, \mathcal{T}\rangle \in \mathbf{L C A G}$ and $\mathcal{U}=\mathcal{T}^{+}$?

(2) Let $G$ be an Abelian group with topological group topologies $\mathcal{T}$ and $\mathcal{U}$ such that $\langle G, \mathcal{T}\rangle \in \mathbf{L C A G},\langle G, \mathcal{U}\rangle \in \mathbf{T B A G}, \mathcal{U} \subseteq \mathcal{T}$, and each $A \subseteq G$ satisfies: $A$ is $\mathcal{T}$-compact if and only if $A$ is $\mathcal{U}$-compact. Does it follow that $\mathcal{U}=\mathcal{T}^{+}$?

We show in this paper that the answer to both these questions is "No". In what follows all hypothesized topological groups are assumed to satisfy the Hausdorff separation axiom. As is well-known, this ensures that our topological groups are completely regular, Hausdorff spaces, i.e., Tikhonov spaces.

The next two theorems record and assemble certain familiar facts from the literature. These will be used below.

1.3. TheOrem. Let $G=\langle G, \mathcal{T}\rangle$ be a topological group.

(a) If $G$ is first countable then $G$ is metrizable.

(b) If $G$ has a dense metrizable subgroup then $G$ itself is metrizable.

(c) If $G$ is Abelian and $G \in \mathbf{M A P}$, then $\langle G, \mathcal{T}\rangle^{\wedge}=\left\langle G, \mathcal{T}^{+}\right\rangle^{\wedge}$.

(d) If $N$ is a compact subgroup of a topological group $G$, and if $\{x N$ : $x \in A\}$ is compact in $G / N$ (with $A \subseteq G$ ), then $A N$ is compact in $G$.

A proof of (a) is given in $[\mathrm{HR}](8.3)$; (b) follows from (a) and the fact that if $p \in D \subseteq X$ with $D$ dense in $X$ and $X$ a Tikhonov space, then the local weight of $D$ at $p$ is equal to the local weight of $X$ at $p$. As for (c), the inclusion $\subseteq$ is clear from the definitions of $\mathrm{b} G$ and $\mathcal{T}^{+}$, while $\supseteq$ follows from the inclusion $\mathcal{T}^{+} \subseteq \mathcal{T}$.

Statement $(\mathrm{d})$ appears in $[\mathrm{HR}](5.24(\mathrm{a}))$.

1.4. Theorem. Let $G, H \in \mathbf{L C A G}$. Then

(a) $\mathrm{b}(G \times H)=\mathrm{b} G \times \mathrm{b} H$

(b) every subgroup $F$ of $G$ satisfies $F \in \mathbf{M A P}$ and $\mathrm{b} F=\mathrm{cl}_{\mathrm{b} G} F$; and

(c) if $H \subseteq G$, then $\mathrm{b}(G / H)=\mathrm{b} G / \mathrm{b} H$.

Proof. (a) is immediate from the relation $(G \times H)^{\wedge}=\widehat{G} \times \widehat{H}(\mathrm{cf}$. $[\mathrm{HR}](23.18))$.

(b) That $F \in \mathbf{M A P}$ follows from $G \in \mathbf{M A P}$. Since every $f \in \widehat{F}$ is uniformly continuous on $F$, such $f$ extends continuously over $\mathrm{cl}_{G} F$ and 
hence to $\bar{f} \in \widehat{G}$ ([HR](24.12)); thus $\widehat{G}$ and $\widehat{F}$ induce the same topology on $F$ and (b) follows from Weil's uniqueness theorem cited in 1.1.

(c) The group $H$ is closed in $G([\mathrm{HR}](5.11))$, so it is enough to notice that $\mathrm{b} G / \mathrm{b} H$ is a compact group containing densely an isomorph of $G / H$ (given by $x H \rightarrow x \mathrm{~b} H$ for $x \in G$ ) such that every $h \in(G / H)^{\wedge}$ remains continuous in the topology induced by $\mathrm{b} G / \mathrm{b} H$ on $G / H$; thus $\mathrm{b} G / \mathrm{b} H$ is the Weil completion of $(G / H)^{+}$, and (c) follows.

The following statement is proved by Flor $[\mathrm{F}]$; see also Reid $[\mathrm{Re}]$ and Ross [Ro].

1.5. Theorem. Let $G \in \mathbf{L C A G}$ and let $\lim _{k \rightarrow \infty} x_{k}=p \in \mathrm{b} G$ with each $x_{k} \in G$. Then

(a) $p \in G$, and

(b) not only $x_{k} \rightarrow p\left(\mathcal{T}^{+}\right)$, but also $x_{k} \rightarrow p(\mathcal{T})$.

1.6. Notation. When we consider the additive group $\mathbb{R}$ or its subgroups $\mathbb{Z}$ and $\mathbb{Q}$, we use additive notation and we denote the neutral element by the symbol 0 . In other contexts in this paper, even when the group in question is assumed Abelian, we use multiplicative notation and we denote the neutral element by the symbol 1 .

1.7. Remarks. (a) The map $e: G \rightarrow \mathrm{b} G$ is an isomorphism (since we have defined bG only when $G$ is Abelian and $\widehat{G}$ separates points of $G$ ) but it is to be noted that only in the most trivial of cases is the group $\mathrm{b} G$ a compactification of $G$ in the topologist's usual sense. Indeed, according to $[\mathrm{CR}](1.2)$ the isomorphism $e$ is a homeomorphism from $G=\langle G, \mathcal{T}\rangle$ into bG if and only if $G \in$ TBAG; for $G \in$ LCAG this occurs if and only if $G$ is compact (in which case $e$ is a homeomorphism of $\langle G, \mathcal{T}\rangle$ onto $\mathrm{b} G$ ).

(b) There follows immediately from 1.5 the well-known fact that (except in the case that $G$ is already a compact metric group) the groups b $G$ with $G \in \mathbf{L C A G}$ are never metrizable. For $G^{+}$is dense in $\mathrm{b} G$, so if $\mathrm{b} G$ is metrizable then for every $p \in \mathrm{b} G \backslash G$ there is $x_{k} \in G$ such that $x_{k} \rightarrow p$.

(c) Theorem 1.5(a) is the statement that for $G \in$ LCAG no sequence

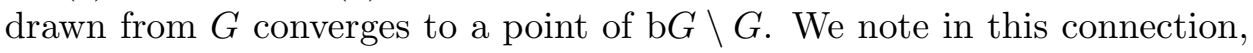
however, that the space $\mathrm{b} G$ does admit non-trivial convergent sequences. Indeed, $\mathrm{b} G$, like every compact group, is a dyadic space [Vi], [K]; therefore, except in the trivial case that $G=\mathrm{b} G$ with $|G|<\omega$, every point of $\mathrm{b} G$ is the limit of a non-trivial sequence. (For a related proof one may use the fact $[\check{\mathrm{S}}]$, [Sh] that $\mathrm{b} G \supseteq\{ \pm 1\}^{\omega}$ when $|G| \geq \omega$, so some - hence, every-point of $\mathrm{b} G$ is the limit of a non-trivial sequence.)

(d) The proof in $[G]$ of Glicksberg's theorem proceeds by way of the Riemann-Lebesgue lemma and a theorem of Grothendieck concerning $C^{*}$ algebras and Radon measures. For a direct, succinct, and "natural" proof 
of Glicksberg's theorem, the reader may consult [T1], [T2], [CT], [T3]; see also [Re], [F], [DPS] (3.4.3), [Hu], [Mo].

(e) Some of the results of this paper were announced in [CHR].

1.8. Acknowledgements. We have profited substantially and in many ways from an unusually creative referee's report. In addition to suggestions about spelling, punctuation, grammar and exposition, which are incorporated into the manuscript without comment, the referee has contributed several helpful and interesting mathematical questions and he/she has shortened and simplified at least one of our proofs; these are acknowledged in situ.

2. LCA groups strongly respect compactness. Throughout this section for $G \in \mathbf{M A P}$ and for $N$ a closed subgroup of bG, we denote by $\pi$ the canonical homomorphism from $\mathrm{b} G$ onto $\mathrm{b} G / N$ and by $\phi$ the restriction function $\phi=\pi \mid G$. (Strictly speaking, we have $\phi=\pi \circ e$ with $e: G \rightarrow \mathrm{b} G$ defined as in 1.1.) We use the symbols $\pi$ and $\phi$ only in this context.

2.1. Definition. Let $G \in \mathbf{M A P}$ and let $N$ be a closed subgroup of bG. Then

(a) $N$ preserves compactness provided: If $A \subseteq G$, then $\phi[A]$ is compact in bG/N if and only if $A \cdot(N \cap G)$ is compact in $G$; and

(b) $G$ strongly respects compactness if every closed, metrizable subgroup of $\mathrm{b} G$ preserves compactness.

2.2. Remarks. (a) The metrizability hypothesis in Definition 2.1(b) may appear artificial; the reader may be tempted, as were the authors, to conjecture that every compact (not necessarily metrizable) subgroup $N$ of $\mathrm{b} G$ preserves compactness. We show in Example 3.2, however, that this proposed generalization of 2.10 can fail, even when $N \cap G=\{1\}$. (Theorem 1.3(d) shows that the metrizability hypothesis for $N$ is superfluous in the case that $N \subseteq G$.) In the positive direction, Example 3.8 shows that there can exist non-metrizable subgroups $N$ of $\mathrm{b} G$ which preserve compactness. For an extension of Theorem 2.10 to discrete groups, see 3.11 below.

(b) Our principal result, Theorem 2.10, shows that every $G \in$ LCAG strongly respects compactness. By way of motivation let us note that this statement generalizes Glicksberg's theorem. Indeed, the special case $N=$ $\{1\}$ of 2.10 asserts that $\left\langle A, \mathcal{T}^{+}\right\rangle=\phi[A] \subseteq \mathrm{b} G$ is compact if and only if $\langle A, \mathcal{T}\rangle=A \cdot\{1\} \subseteq G$ is compact.

(c) The referee has contributed a nifty argument which simplified the proof of one of our principal lemmas, namely 2.6. It is interesting to note an unexpected by-product of the referee's argument: the new proof of our generalization of Glicksberg's theorem, unlike the old, requires and invokes Glicksberg's original theorem not in its full generality but (only) for $\sigma$ compact, metrizable groups $G \in \mathbf{L C A G}$. 
(d) In the lemmas and theorems of this section, we show that various MAP groups $G$ strongly respect compactness. In each case it is required to show (for suitable $A \subseteq G$ and $N \subseteq \mathrm{b} G$ ) that $\phi[A]=\{a N: a \in A\}$ is compact in bG/N if and only if $A \cdot(N \cap G)$ is compact in $G$. Since $\phi$ is continuous from $G$ into $\mathrm{b} G / N$ and $\phi[A]=\phi[A \cdot(N \cap G)]$, the "if" implication is trivial in each case; accordingly in these proofs we restrict our attention to the "only if" implications.

2.3. Lemma. Let $G_{0}$ and $G_{1}$ be MAP groups which strongly respect compactness, and let $G=G_{0} \times G_{1}$. Then $G$ strongly respects compactness.

Pro of. Let $N$ be a compact, metrizable subgroup of bG= $\mathrm{b} G_{0} \times \mathrm{b} G_{1}$, and let $A \subseteq G$ have the property that $\phi[A]=\{x N: x \in A\}$ is a compact subset of bG/N. We show that $A \cdot(N \cap G)$ is compact in $G$.

Let $\varrho_{i}$ denote the natural projection

$$
\varrho_{i}: \mathrm{b} G \rightarrow \mathrm{b} G_{i} \quad(i=1,2)
$$

and set $A_{i}=\varrho_{i}[A]$ and $N_{i}=\varrho_{i}[N]$. Since $\phi[A]$ is compact and $\pi: \mathrm{b} G \rightarrow$ $\mathrm{b} G / N$ is continuous, the set $\pi^{-1}(\phi[A])$, which is $A N$, is compact in $\mathrm{b} G$; hence $\pi_{i}\left[\varrho_{i}[A N]\right]$ is compact in $\mathrm{b} G_{i} / N_{i}$. It is clear that $\pi_{i}\left[\varrho_{i}[A N]\right]=\phi_{i}\left[A_{i}\right]$. (Indeed, if $a=\left\langle a_{1}, a_{2}\right\rangle \in A$ and $n=\left\langle n_{1}, n_{2}\right\rangle \in N$ then

$$
\begin{aligned}
\pi_{i}\left(\varrho_{i}(\text { an })\right) & =\pi_{i} \varrho_{i}\left(\left\langle a_{1} n_{1}, a_{2} n_{2}\right\rangle\right) \\
& =a_{i} n_{i} N_{i}=a_{i} N_{i}=\phi_{i}\left(a_{i}\right) \quad \text { with } a_{i} \in A_{i} ;
\end{aligned}
$$

and if $a_{i} \in A_{i}$ then there is $x \in A \subseteq A N$ such that $a_{i}=\varrho_{i}(x)$ and we have

$$
\left.\phi_{i}\left(a_{i}\right)=a_{i} N_{i}=\pi_{i}\left(\varrho_{i}(x)\right) \in \pi_{i} \varrho_{i}[A N] .\right)
$$

Since $\phi_{i}\left[A_{i}\right]$ is compact in $\mathrm{b} G_{i} / N_{i}, N_{i}$ is metrizable, and $G_{i}$ strongly respects compactness, it follows that the space $A_{i} \cdot\left(G_{i} \cap N_{i}\right)$ is compact in $G_{i}$. Writing $B_{i}=A_{i} \cdot\left(G_{i} \cap N_{i}\right)$ and $B=B_{0} \times B_{1}$ we have: $B$ is compact in $G$.

We claim finally that $A \cdot(N \cap G) \subseteq B$. Indeed, if $a=\left\langle a_{0}, a_{1}\right\rangle \in A$ and $n=\left\langle n_{0}, n_{1}\right\rangle \in N \cap G$ then $a_{i} \in \varrho_{i}[A]$ and $n_{i} \in \varrho_{i}[N] \cap G_{i}=N_{i} \cap G_{i}$, so $(\text { an })_{i}=a_{i} n_{i} \in B_{i}$.

Since $A N$ is compact the set $A N \cap G$, which is $A \cdot(N \cap G)$, is closed in $G$. Since $A \cdot(N \cap G) \subseteq B$ and $B$ is compact the set $A \cdot(N \cap G)$ is compact in $G$, as required.

2.4. LemmA. Every compact Abelian group strongly respects compactness.

Proof. This is immediate from Theorem 1.3(d).

2.5. Lemma. Let $G=\langle G, \mathcal{T}\rangle \in \mathbf{L C A G}$, and let $N$ be a closed, metrizable subgroup of $\mathrm{b} G$, and set $G \cap N=H$. Then the spaces $\langle H, \mathcal{T}\rangle$ and $\left\langle H, \mathcal{T}^{+}\right\rangle$are compact, metrizable, and homeomorphic. 
First proof (using Glicksberg's theorem). It suffices to show that $\mathrm{H}^{+}$is compact and metrizable. Clearly $\mathrm{H}^{+}$is metrizable. To see that $\mathrm{H}^{+}$ is closed in $N=\left\langle N, \mathcal{T}^{+}\right\rangle$, note that if $x_{k} \in H^{+}$and $x_{k} \rightarrow p \in N$ then $p \in H^{+}$by Theorem $1.5(\mathrm{a})$.

Second proof (not using Glicksberg's theorem). It suffices to show that $H$ is compact and metrizable. Since $H^{+}$is closed in $G^{+}$and $e: G \rightarrow G^{+}$ is continuous, the space $e^{-1}\left(H^{+}\right)=H$ is closed in $G=\langle G, \mathcal{T}\rangle$ so $H=$ $\langle H, \mathcal{T}\rangle \in$ LCAG. It follows from Theorem 1.4(b) that the Bohr topology of $H$ is the topology which $H$ inherits from $G^{+}$; thus $H^{+}$, a subspace of $N$, is metrizable. Since $H^{+}$is a dense topological subgroup of $\mathrm{b} H$, the compact group $\mathrm{b} H$ is itself metrizable (Theorem 1.3(b) above). That $H$ is compact (i.e., $H=\mathrm{b} H$ ) now follows from Remark 1.7(b).

We say as usual in the following proof that a family $\mathcal{N}$ of (not necessarily open) subsets of a space $X$ is a network for $X$ if for every neighborhood $U$ of $x \in X$ there is $F \in \mathcal{N}$ such that $x \in F \subseteq U$.

2.6. Lemma. Let $G \in \mathbf{L C A G}$ with $G$ metrizable and $\sigma$-compact. Then $G$ strongly respects compactness.

Pr o of. Let $N$ be a compact, metrizable subgroup of bG and let $A \subseteq G$ have the property that $\phi[A]=\{x N: x \in A\}$ is compact in $\mathrm{b} G / N$. We must show that $A \cdot(N \cap G)$ is compact in $G$.

Both $A$ and $N$ are separable and metrizable, so each has a countable network. Thus $A \times N$, hence its continuous image $A N$, has a countable network. Now $A N=\pi^{-1}(\phi[A])$ is compact in $\mathrm{b} G$, so $A N$ is metrizable. (Here we use the case $\kappa=\omega$ of the following familiar theorem: a compact space with a network of cardinality $\kappa$ has weight $\kappa$. See $[E](3.1 .19)$ for a proof of this statement.)

To show that $A \cdot(N \cap G)$ is compact in $G$ it is enough, since $G$ respects compactness, to show that $A \cdot(N \cap G)$ is compact in $G^{+}$; for that it suffices to show that $A \cdot(N \cap G)$ is closed in the compact metrizable space $A N$. Let $x_{k} \rightarrow p \in A N \subseteq \mathrm{b} G$ with $x_{k} \in A \cdot(N \cap G)=A N \cap G$. Then $p \in G$ by 1.5 above and we have $p \in A N \cap G=A \cdot(N \cap G)$, as required.

2.7. Remark. In the original version of this manuscript we deduced the metrizability of $A N$ in 2.6 from Glicksberg's theorem and this result of Smirnov [Sm] (cf. also A. H. Stone [Sto]): A compact space which is the union of countably many closed metrizable spaces is itself metrizable. Indeed, $(A N) \cap G$ has the form $\bigcup_{n<\omega} K_{n}$ with $K_{n}$ metrizable (in $G^{+}$, hence in $G$ by Glicksberg's theorem), so $A N=\bigcup_{n<\omega}\left(K_{n} \cdot N\right)$ with $K_{n} \cdot N$ compact and metrizable. 
Every locally compact topological group contains an open-and-closed compactly generated subgroup. On the basis of the lemmas above we are able to show that these (Abelian) topological groups strongly respect compactness.

2.8. TheOREM. Let $G$ be a locally compact, compactly generated, Abelian topological group. Then $G$ strongly respects compactness.

Proof. The group $G$ is topologically isomorphic to a group of the form $\mathbb{R}^{n} \times \mathbb{Z}^{k} \times F$ for integers $n, k \geq 0$ and $F$ a compact group (cf. [HR](9.8)). Since $\mathbb{R}^{n} \times \mathbb{Z}^{k}$ strongly respects compactness by Lemma 2.6 , and $F$ strongly respects compactness by Lemma 2.4 , the conclusion follows from Lemma 2.3 .

2.9. Lemma. Let $G$ be a discrete Abelian group. Then $G$ strongly respects compactness.

Pro of. Let $N$ be a closed, metrizable subgroup of bG and let $A \subseteq G$ satisfy: $\phi[A]$ is compact in $\mathrm{b} G / N$. Suppose that $|A| \geq \omega$, choose $A^{\prime} \subseteq A$ such that $\left|A^{\prime}\right|=\omega$, let $G_{0}$ be the subgroup of $G$ generated by $A^{\prime}$, and set $A_{0}=G_{0} \cap A$ and $N_{0}=N \cap \mathrm{b} G_{0}$. Then $N_{0}$ is a closed, metrizable subgroup of $\mathrm{b} G_{0}$.

The groups $\mathrm{b} G_{0} / N_{0}$ and $\pi\left[\mathrm{b} G_{0}\right]$ (a subgroup of $\pi[\mathrm{b} G]=\mathrm{b} G / N$ ) are topologically isomorphic groups under a correspondence taking $\phi_{0}\left[A_{0}\right]$ onto $\phi[A] \cap \pi\left[\mathrm{b} G_{0}\right]$ (cf. $[\mathrm{HR}]\left(5.31\right.$ and 5.33)). Thus $\phi_{0}\left[A_{0}\right]$ is compact in b $G_{0} / N_{0}$. Since $G_{0}$ strongly respects compactness (by Lemma 2.6) the space $A_{0} \cdot\left(N_{0} \cap\right.$ $\left.G_{0}\right)$ is compact in $G_{0}$ and hence finite. Thus $A_{0}$ itself is finite, contrary to the conditions $A_{0} \supseteq A^{\prime},\left|A^{\prime}\right|=\omega$.

We turn now to the principal positive result of this paper.

2.10. TheOREM. Let $G \in \mathbf{L C A G}$. Then $G$ strongly respects compactness.

Pr o of. Let $N$ be a compact, metrizable subgroup of bG and let $A \subseteq G$ satisfy: $\phi[A]$ is compact in $\mathrm{b} G / N$.

Since (according to Lemma 2.5) the group $G \cap N$ is compact, there is an open-and-closed, compactly generated subgroup $H$ of $G$ such that $G \cap N \subseteq H$. We choose such $H$ and we note from 1.4 that $\mathrm{b} H=\mathrm{cl}_{\mathrm{b} G} H$ and that $\mathrm{b}(G / H)$ is (topologically isomorphic to) $\mathrm{b} G / \mathrm{b} H$. Denoting by $\varrho: \mathrm{b} G \rightarrow \mathrm{b} G / \mathrm{b} H$ the canonical homomorphism, we note that the natural $\operatorname{maps} q: \mathrm{b} G / N \rightarrow \mathrm{b} G /(N \cdot \mathrm{b} H)$ and

$$
\psi: \mathrm{b} G / \mathrm{b} H \rightarrow(\mathrm{b} G / \mathrm{b} H) / \varrho[N] \approx \mathrm{b} G /(N \cdot \mathrm{b} H)
$$

satisfy $q \circ \phi=\psi \circ \varrho \mid G$; indeed, $q \circ \pi=\psi \circ \varrho$, so $\psi[\varrho[A]]=q[\phi[A]]$ is compact in $(\mathrm{b} G / \mathrm{b} H) / \varrho[N]$. (The indicated isomorphism $(\mathrm{b} G / \mathrm{b} H) / \varrho[N] \approx$ $\mathrm{b} G /(N \cdot \mathrm{b} H)$ is given by $[\mathrm{HR}](5.35)$.) Since $\varrho[N]$ is a compact, metrizable 
subgroup of $\mathrm{b} G / \mathrm{b} H=\mathrm{b}(G / H)$ and $G / H$ is a discrete Abelian group, the set $\varrho[A] \cdot(G / H \cap \varrho[N])$ is compact in $G / H$ by Lemma 2.9. It then follows that $\varrho[A]$ is finite, i.e., that $A$ meets only finitely many cosets of $H$ in $G$. Replacing $H$ if necessary by the subgroup of $G$ generated by $H \cup A$, we assume in what follows that $A \subseteq H$. The inclusion $G \cap N \subseteq H$ perseveres, so that $G \cap N=H \cap N$.

Now let $\sigma: \mathrm{b} G \rightarrow \mathrm{b} G /(N \cap \mathrm{b} H)$ and $g: \mathrm{b} G /(N \cap \mathrm{b} H) \rightarrow \mathrm{b} G / N$ be the (continuous) homomorphisms defined by

$$
\begin{gathered}
\sigma(p)=p(N \cap \mathrm{b} H), \\
g(p(N \cap \mathrm{b} H))=p N .
\end{gathered}
$$

Since $\phi[A]=\{a N: a \in A\}$ is compact in $\mathrm{b} G / N$ the space

$$
g^{-1}(\phi[A])=\{p(N \cap \mathrm{b} H): p \in \mathrm{b} G, \text { there is } a \in A \text { such that } p N=a N\}
$$

is compact in $\mathrm{b} G /(N \cap \mathrm{b} H)$. That is, writing

$$
\begin{aligned}
& Y=\left\{p(N \cap \mathrm{b} H): p \in \mathrm{b} G \text { and there are } p^{\prime} \in \mathrm{b} H, a \in A\right. \text { such that } \\
& \left.\qquad p(N \cap \mathrm{b} H)=p^{\prime}(N \cap \mathrm{b} H) \text { and } p N=a N\right\},
\end{aligned}
$$

we have: $Y$ is compact in $\mathrm{b} G /(N \cap \mathrm{b} H)$. Now if $p(N \cap \mathrm{b} H) \in Y$ then $p \in \mathrm{b} H$ (since $p \mathrm{~b} H=p^{\prime} \mathrm{b} H$ for some $p^{\prime} \in \mathrm{b} H$ ). Thus

$$
Y=\{p(N \cap \mathrm{b} H): p \in \mathrm{b} H, \text { there is } a \in A \text { such that } p N=a N\} .
$$

It then follows, denoting by $\pi^{\prime}: \mathrm{b} H \rightarrow \mathrm{b} H /(N \cap \mathrm{b} H)$ the canonical homomorphism, that $Y=\pi^{\prime}[A]$. (Indeed, if $p(N \cap \mathrm{b} H) \in Y$ with $p N=a N$ then from $p \in \mathrm{b} H$ and $A \subseteq H \subseteq \mathrm{b} H$ follows

$$
p(N \cap \mathrm{b} H)=a(N \cap \mathrm{b} H)=\pi^{\prime}[a] \in \pi^{\prime}[A] ;
$$

the inclusion $\pi^{\prime}[A] \subseteq Y$ is immediate from $A \subseteq H \subseteq \mathrm{b} H$.)

Since $H$ is locally compact and compactly generated and $A$ is a subset of $H$ such that $\{a(N \cap \mathrm{b} H): a \in A\}$ is compact (with $N \cap \mathrm{b} H$ a compact metrizable subgroup of $\mathrm{b} H)$, it follows from Theorem 2.8 that $A \cdot(N \cap \mathrm{b} H \cap$ $H)$ is compact in bH. From $N \cap H=N \cap G$ it follows that $A \cdot(N \cap G)$ is compact in $\mathrm{b} H$, hence in $\mathrm{b} G$, as required.

2.11. Corollary. Let $G=\langle G, \mathcal{T}\rangle \in \mathbf{L C A G}$ and let $N$ be a compact, metrizable subgroup of $\mathrm{b} G$. If $N \cap G=\{1\}$, then the following conditions are equivalent.

(a) $A$ is compact in $\langle G, \mathcal{T}\rangle$;

(b) $A$ is compact in $\left\langle G, \mathcal{T}^{+}\right\rangle$;

(c) $\phi[A]$ is compact in $\mathrm{b} G / N$.

Pr o of. The equivalence $(\mathrm{a}) \Leftrightarrow(\mathrm{b})$ is Glicksberg's theorem, restated here for emphasis, while $(\mathrm{a}) \Leftrightarrow(\mathrm{c})$ is the case $N \cap G=\{1\}$ of Theorem 2.10. 
2.12. Discussion. (a) The referee has raised the following question: If $N$ is a compact, metrizable subgroup of a compact Abelian group $G$, and if $A \subseteq G$ satisfies $\langle A\rangle \cap N=\{1\}$ (with $\langle A\rangle$ the subgroup of $G$ generated by $A$ ) with $A N$ closed in $G$, must $A$ itself be closed in $G$ ? This question is relevant to the present enterprise, since a positive answer could greatly simplify the proof of Theorem 2.10. It is interesting to see that the answer to this question is "No". Let $G=\mathbb{T}$ and $N=\{+1,-1\} \subseteq \mathbb{T}$ and, with $x_{n}$ a sequence in the Prüfer 3 -group $\mathbb{Z}\left(3^{\infty}\right)$ such that $x_{n} \rightarrow-1$, define $A=$ $\left\{x_{n}: n<\omega\right\} \cup\{1\}$. Evidently $A$ is not compact but $A N$, the union of two converging sequences and their limit points, is compact. From $A \subseteq \mathbb{Z}\left(3^{\infty}\right)$ and $\mathbb{Z}\left(3^{\infty}\right) \cap N=\{1\}$ follows $\langle A\rangle \cap N=\{1\}$, as required.

(b) The referee further has raised the question whether Theorem 2.10 characterizes those Abelian groups which are locally compact. In detail: If $G \in$ MAP and $G$ strongly respects compactness, must $G$ be locally compact? The answer to this question is "No"; the additive group $G=\mathbb{Q}$ is an example. Surely $\mathbb{Q}$ is not locally compact. Now bQQ $=$ b $\mathbb{R}$ by $1.4(\mathrm{~b})$, and if $N$ is a closed, metrizable subgroup of b $\mathbb{Q}$ then 2.5 guarantees that $N \cap \mathbb{R}$ is compact (so $\{0\}=N \cap \mathbb{Q}=N \cap \mathbb{R}$ ). Suppose now that $A \subseteq \mathbb{Q}$ and $\phi: \mathrm{b} \mathbb{Q} \rightarrow \mathrm{b} \mathbb{Q} / N$ satisfy: $\phi[A]$ is compact in $\mathrm{b} \mathbb{Q} / N$. Since $\mathbb{R}$ strongly respects compactness we conclude that $A+\{0\}=A+(N \cap \mathbb{R})$ is compact in $\mathbb{R}$, so $A$ is compact in $\mathbb{R}$ and hence in $\mathbb{Q}$.

\section{Examples and counterexamples}

3.1. R e mark. One may be tempted to strengthen Theorem 2.10 to the statement that every $G \in$ MAP strongly respects compactness, but this result fails. Indeed, it is shown in [RT], correcting a statement from [Ve], that there are (Abelian) groups which satisfy Pontryagin duality but which do not respect compactness.

3.2. Examples. (a) The condition in Theorem 2.10 that $N$ be metrizable cannot be omitted. Let $G$ be any locally compact Abelian group with a closed, non-compact subgroup $H$ of finite index (for example, $G$ is noncompact and $G=H$ ) and take $N=\mathrm{b} H=\operatorname{cl}_{\mathrm{b} G} H$. Since $G / H$ is finite and

$$
\mathrm{b} G / N=\mathrm{b} G / \mathrm{b} H=\mathrm{b}(G / H)=G / H
$$

by Theorem 1.4 above, every non-empty subset $A$ of $G$ satisfies: $\phi[A]$ is finite and hence compact. But $A \cdot(N \cap G)=A H$ is not compact, since $A H$ contains a homeomorph of the non-compact space $H$ as a closed subspace.

(b) It is more challenging to show, as promised in 2.2(a), that there exist $G \in$ LCAG and a closed (non-metrizable) subgroup $N$ of b $G$ such that $N \cap G=\{1\}$ and $N$ does not preserve compactness. For an example to this effect let $G=\mathbb{Z}$ in the usual (discrete) topology, choose $\alpha \in[0,1] \backslash \mathbb{Q}$, define 
$\chi \in \widehat{\mathbb{Z}}$ by $\chi(n)=e^{2 \pi i \alpha n}$, let $h$ be the continuous homomorphism from $\mathrm{b} \mathbb{Z}$ to $\mathbb{T}$ such that $\chi \subseteq h$, let $F$ be any finite subgroup of $\mathbb{T}$ (e.g., $F=\{1\}$ ), and set $N=h^{-1}(F) \subseteq \mathrm{b} \mathbb{Z}$. Now $F$ is a closed $\mathrm{G}_{\delta}$-subset of $\mathbb{T}$, so $N$ is a compact, $\mathrm{G}_{\delta}$-subgroup of $\mathrm{b} \mathbb{Z}$ such that $N \cap \mathbb{Z}=\{0\}$; that the quotient group $\mathrm{b} \mathbb{Z} / N$ is metrizable is given by $[\mathrm{HR}](8.6)$.

Since $\phi[\mathbb{Z}]$ is dense in $\mathrm{b} \mathbb{Z} / N$ and this metrizable quotient space is not discrete, there is a sequence $\left\{n_{k}: k<\omega\right\} \subseteq \mathbb{Z} \backslash\{0\}$ such that $\phi\left(n_{k}\right) \rightarrow$ $\phi(0)=N$ in $\mathrm{b} \mathbb{Z} / N$. It is then clear, writing

$$
A=\left\{n_{k}: k<\omega\right\} \cup\{0\} \subseteq \mathbb{Z},
$$

that $\phi[A]$ is compact in $\mathrm{b} \mathbb{Z} / N$, while $A$ is not compact in the discrete space $\mathbb{Z}$.

It is reassuring to observe in the example just given that the compact group $N$ is not metrizable - that is, this example does not contradict Theorem 2.10. (If $N$ were metrizable then both $N$ and $\mathrm{b} \mathbb{Z} / N$ would have cardinality $\leq \mathbf{c}$, so $|\mathrm{b} \mathbb{Z}| \leq \mathbf{c}$; but every infinite compact group $K$ satisfies $|K|=2^{\mathrm{w} K}$, so from $\mathrm{w}(\mathrm{b} \mathbb{Z})=|\widehat{\mathrm{b} Z}|=|\widehat{\mathbb{Z}}|=\mathbf{c}$ follows $|\mathrm{b} \mathbb{Z}|=2^{\mathbf{c}}$. Alternatively, one may argue that if $\{0\}$ is a $\mathrm{G}_{\delta}$-subset of $N$ then $\{0\}$ would be a $\mathrm{G}_{\delta}$-subset of $\mathrm{b} \mathbb{Z}$, so $\mathrm{b} \mathbb{Z}$ would be metrizable; but $\mathbb{Z}$ is dense in $b \mathbb{Z}$ so if $\mathrm{b} \mathbb{Z}$ were metrizable then every point of $\mathrm{b} \mathbb{Z}$ is the limit of a sequence drawn from $\mathbb{Z}$, contrary to Theorem 1.5.)

3.3. Re mark. It is easy to find examples of totally bounded Abelian groups $\langle G, \mathcal{U}\rangle$ such that $\mathcal{U}=\mathcal{T}^{+}$for no locally compact topological group topology $\mathcal{T}$ on $G$. Indeed, according to Remark 1.7(b) the topologies $\mathcal{T}^{+}$ with $\mathcal{T}$ locally compact are seldom metrizable, so it is enough to choose $G=\langle G, \mathcal{U}\rangle \in$ TBAG with $G$ metrizable and non-compact (for example, use $G=\mathbb{Q} / \mathbb{Z})$. More generally one may choose any totally bounded Abelian group $\langle G, \mathcal{U}\rangle$ with a closed, metrizable non-compact subgroup $H$; for if $\mathcal{U}=\mathcal{T}^{+}$with $\langle G, \mathcal{T}\rangle \in \mathbf{L C A G}$, then $\langle H, \mathcal{T}\rangle=e^{-1}(H)$ is closed in $\langle G, \mathcal{T}\rangle$ so $\langle\mathrm{b} H, \mathcal{T}\rangle=\mathrm{cl}_{\mathrm{b} G} H$ is metrizable by $1.3(\mathrm{~b})$ and $1.4(\mathrm{~b})$. Thus, replacing $G=\mathbb{Q} / \mathbb{Z}$ by $G^{\alpha}$ for cardinals $\alpha \geq \omega$, one obtains examples of arbitrarily large weight and cardinality.

3.4. For a (Hausdorff) space $X=\langle X, \mathcal{T}\rangle$ we denote as usual by $\mathrm{k} X$ or by $\langle X, \mathrm{k} \mathcal{T}\rangle$ the set $X$ with the topology $\mathrm{k} \mathcal{T}$ defined as follows: A subset $U$ of $X$ is $\mathrm{k} \mathcal{T}$-open if and only if $U \cap K$ is (relatively) $\mathcal{T}$-open in $K$ for every $\mathcal{T}$-compact subset $K$ of $X$. Then $\mathrm{k} X$ is a $\mathrm{k}$-space (that is, $\mathrm{k} X=\mathrm{kk} X$ ), $\mathrm{k} \mathcal{T}$ is the smallest $\mathrm{k}$-space topology on $X$ containing $\mathcal{T}$, and $\mathrm{k} \mathcal{T}$ is the unique $\mathrm{k}$-space topology for $\langle X, \mathcal{T}\rangle$ such that $\mathrm{k} \mathcal{T} \supseteq \mathcal{T}$ and the $\mathrm{k} \mathcal{T}$-compact sets are exactly the $\mathcal{T}$-compact sets. For proofs of these facts and additional commentary the reader may consult [E](pp. 201-204).

The following simple observation is taken from [T1](6.24). 
3.5. Theorem. Let $G=\langle G, \mathcal{T}\rangle \in$ LCAG. Then $G=\mathrm{k} G^{+}$(more formally: $\left.\langle G, \mathcal{T}\rangle=\mathrm{k}\left(\left\langle G, \mathcal{T}^{+}\right\rangle\right)\right)$.

Pro of. Since $\mathcal{T} \supseteq \mathcal{T}^{+}$and $\mathcal{T}$ is a k-space topology for $G$ such that the $\mathcal{T}$-compact subsets are exactly the $\mathcal{T}^{+}$-compact sets, the result follows from the uniqueness theorem cited in 3.4.

It has been shown by LaMartin [L] that for $\alpha>\omega$ and $G=\mathbb{R}^{\alpha}$ the space $\mathrm{k} G$ is not a topological group (indeed, $\mathrm{k} G$ is a Hausdorff non-regular topological space). As noticed in [T3](1.14) this furnishes the following observations.

3.6. TheOREM. Let $\alpha>\omega$. Then

(a) $\mathrm{k}\left(\left(\mathbb{R}^{+}\right)^{\alpha}\right)=\mathrm{k}\left(\mathbb{R}^{\alpha}\right)$;

(b) $\mathrm{k}\left(\left(\mathbb{R}^{+}\right)^{\alpha}\right)$ is not a topological group;

(c) there is no locally compact topological group topology $\mathcal{T}$ on $\mathbb{R}^{\alpha}$ for which the totally bounded topological group $\left(\mathbb{R}^{+}\right)^{\alpha}$ satisfies $\left(\mathbb{R}^{+}\right)^{\alpha}=$ $\left\langle\mathbb{R}^{\alpha}, \mathcal{T}\right\rangle^{+}$.

Proof. Statement (a) follows from the fact that $\mathbb{R}^{\alpha}$ and $\left(\mathbb{R}^{+}\right)^{\alpha}$ have the same compact sets, while the topology of the former contains that of the latter; (b) is then immediate from LaMartin's theorem, and (c) follows from (b) and Theorem 3.5.

3.7. Example. Let $G \in$ TBAG satisfy $\mathrm{k} G \in$ LCAG. Does it follow that $G=(\mathrm{k} G)^{+}$? This question is closely related to question (2) of 1.2 above: If $\langle G, \mathcal{T}\rangle \in$ LCAG and $\langle G, \mathcal{U}\rangle \in$ TBAG with $\mathcal{U} \subseteq \mathcal{T}$ and the $\mathcal{T}$-compact subsets of $G$ are exactly the $\mathcal{U}$-compact subsets of $G$, does it follow that $\mathcal{U}=\mathcal{T}^{+}$? We show that the answer to both these questions is "No"; in our example the group $G$ is $\mathbb{Z}$ appropriately topologized, and $\mathrm{k} G$ is the discrete group $\mathbb{Z}$. See also in this connection [T3](1.12) and [T1](6.28(a), $6.30)$.

We claim first that there is a non-trivial compact metrizable subgroup $N$ of bZ such that $N \cap \mathbb{Z}=\{0\}$. To see this, first write the (discrete) circle group $\mathbb{T}_{\mathrm{d}}$ in the form $\mathbb{T}_{\mathrm{d}}=A_{\mathrm{d}} \times B_{\mathrm{d}}$ with $A_{\mathrm{d}}$ and $B_{\mathrm{d}}$ subgroups and $\left|A_{\mathrm{d}}\right|=\omega$; see $[\mathrm{HR}](\mathrm{A} .14)$ for the availability of such a decomposition. Now from $\widehat{\mathrm{b} \mathbb{Z}}=\mathbb{T}_{\mathrm{d}}=A_{\mathrm{d}} \times B_{\mathrm{d}}$ follows $\mathrm{b} \mathbb{Z}=\widehat{\mathbb{T}}_{\mathrm{d}}=\widehat{A}_{\mathrm{d}} \times \widehat{B}_{\mathrm{d}}$; we set $N=\widehat{A}_{\mathrm{d}} \times\{1\} \subseteq \mathrm{b} \mathbb{Z}$, so indeed $N$ is a compact, metrizable subgroup of $\mathrm{b} \mathbb{Z}$. If $h \in N \cap \mathbb{Z}$ then $h$ is continuous in the usual topology of $\mathbb{T}$ - that is, not only $h \in \widehat{\mathbb{T}}_{\mathrm{d}}$ but in fact $h \in \mathbb{Z}=\widehat{\mathbb{T}}$ - so from $h \equiv 1$ on $B$ (and the density of $B$ in $\mathbb{T}$ ) follows $h \equiv 1$. Thus $h$ is the identity element of $\mathrm{b} \mathbb{Z}$. The claim is proved.

To complete the construction define $\phi=\pi \mid \mathbb{Z}$ with $\pi: \mathrm{b} \mathbb{Z} \rightarrow \mathrm{b} \mathbb{Z} / N$ as usual and set $G=\phi[\mathbb{Z}]$. Algebraically $\phi$ is an isomorphism, so there is a topology $\mathcal{U}$ on $\mathbb{Z}$ determined by the condition that $\phi:\langle\mathbb{Z}, \mathcal{U}\rangle \rightarrow G \subseteq \mathrm{b} \mathbb{Z} / N$ 
is a homeomorphism. According to Theorem 2.10 the compact subsets of $G$ (that is, of $\langle\mathbb{Z}, \mathcal{U}\rangle$ ) are exactly the finite sets. Thus the usual discrete topology $\mathcal{T}$ on $\mathbb{Z}$ satisfies $\mathcal{U} \subseteq \mathcal{T}$, and a subset of $\mathbb{Z}$ is $\mathcal{U}$-compact if and only if it is $\mathcal{T}$-compact, so we have $\mathrm{k}\langle\mathbb{Z}, \mathcal{U}\rangle=\langle\mathbb{Z}, \mathcal{T}\rangle$. But the relation $\mathcal{U}=\mathcal{T}^{+}$ fails: We have $\left\langle\mathbb{Z}, \mathcal{T}^{+}\right\rangle^{\wedge}=\widehat{\mathrm{b} \mathbb{Z}}=A_{\mathrm{d}} \times B_{\mathrm{d}}$ while $\langle\mathbb{Z}, \mathcal{U}\rangle^{\wedge}=\langle\mathrm{b} \mathbb{Z} / N\rangle^{\wedge}=B_{\mathrm{d}}$ (cf. $[\mathrm{HR}](23.25)$ or $[\mathrm{CR}])$.

3.8. ExAMPLE. There are locally compact Abelian groups $G$ such that bG contains a closed non-metrizable subgroup $N$ which preserves compactness. An easy example can be constructed as follows: Let $K$ be any nonmetrizable compact Abelian group and let $G=K \times \mathbb{Z}$. Let $N_{1}$ denote a non-trivial closed metrizable subgroup of $\mathrm{b} \mathbb{Z}$, and set $N=K \times N_{1}$. Since $\mathrm{b} G=K \times \mathrm{b} \mathbb{Z}(1.4(\mathrm{a}))$ we deduce that $N$ is a closed non-metrizable subgroup of $\mathrm{b} G$. An argument just as in the proof of Lemma 2.3 shows that $N$ preserves compactness. Since $N_{1} \cap \mathbb{Z}=\{0\}$ by 2.5 , we conclude that $K \approx N \cap G$ is a compact non-metrizable subgroup of $G$.

3.9. Discussion. It is much more challenging, however, to find a discrete Abelian group $G$ such that some closed, non-metrizable subgroup of bG preserves compactness. We achieve this in Theorem 3.12 below. As usual, for $G \in \mathbf{L C A G}$ and $N$ a closed subgroup of $G$, we write

$$
\begin{aligned}
\mathbf{A}(\widehat{G}, N) & =\{\chi \in \widehat{G}: N \subseteq \operatorname{ker}(\chi)\} \\
& =\{\chi \in \widehat{G}: \chi(p)=1 \text { for all } p \in N\} .
\end{aligned}
$$

(The set $\mathbf{A}(\widehat{G}, N)$ is called the annihilator of $N$ in $\widehat{G}$.) In particular, the set $\mathbf{A}(\widehat{\mathrm{bG}}, N)$ is defined (and is a subgroup of $\widehat{\mathrm{bG}}$ ) whenever $N$ is a closed subgroup of $\mathrm{b} G$. of $\widehat{G}$.

We approach Theorem 3.12 via two lemmas concerning the Haar measure

3.10. Lemma. Let $G$ be a discrete Abelian group and let $\lambda$ denote the normalized Haar measure of the group $\widehat{G}$. If $\left\{x_{n}\right\}$ is a faithfully indexed sequence in $G$ and

$$
A=\left\{\chi \in \widehat{G}: \chi\left(x_{n}\right) \rightarrow 1\right\},
$$

then $A$ is $\lambda$-measurable in $\widehat{G}$ and $\lambda(A)=0$.

Proof. Appealing to Pontryagin duality, we identify each $x_{n}$ with the continuous homomorphism from $\widehat{G}$ to $\mathbb{T}$ given by

$$
x_{n}(\chi)=\chi\left(x_{n}\right) \text {. }
$$

Writing

$$
A_{n, m}=\left\{\chi \in \widehat{G}:\left|x_{n}(\chi)-1\right| \leq 1 / m\right\}
$$


for $m, n<\omega$ gives

$$
A=\bigcap_{m<\omega} \bigcup_{N \geq m} \bigcap_{n \geq N} A_{n, m}
$$

thus $A$ is an $\mathrm{F}_{\sigma \delta}$ of $\widehat{G}$, hence is $\lambda$-measurable.

Now for $\chi, \psi \in A$ and $n<\omega$ we have

$$
\begin{gathered}
\left|(\chi \psi)\left(x_{n}\right)-1\right| \leq\left|\chi\left(x_{n}\right)-1\right|+\left|\psi\left(x_{n}\right)-1\right| \text { and } \\
\left|\chi^{-1}\left(x_{n}\right)-1\right|=\left|\chi\left(x_{n}\right)-1\right| ;
\end{gathered}
$$

this shows that $A$ is a subgroup of $\widehat{G}$. If $\lambda A>0$ then from the SteinhausWeil theorem it follows that $A$ is open-and-closed in $\widehat{G}$, so that $|\widehat{G} / A|<\omega$. Thus by $[\mathrm{HR}](23.25)$ the group $(\widehat{\widehat{G} / A})$, which is $\mathbf{A}(G, A)$, is finite. But this is impossible: Let $i: A \rightarrow \widehat{G}$ be the inclusion map. Then $\widehat{i}: G \rightarrow \widehat{A}$ satisfies $\hat{i}(g)=g \circ i=g \mid A[\mathrm{HR}](24.37)$. Now let $\Pi: G \rightarrow G / \mathbf{A}(G, A)$ denote the projection map. By $[\mathrm{HR}](23.25)$ the map $\Lambda:(G / \mathbf{A}(G, A))^{\wedge} \rightarrow$ $\mathbf{A}(\widehat{G}, \mathbf{A}(G, A)$ ) (which is just $A[\mathrm{HR}](24.10)$ ) defined by $\Lambda(\chi)=\chi \circ \Pi$ is a topological isomorphism. Let $y_{n}=\Pi\left(x_{n}\right)$. If $\chi \in(G / \mathbf{A}(G, A))^{\wedge}$, then $\chi\left(y_{n}\right)=\chi \circ \Pi\left(x_{n}\right) \rightarrow 1$ by definition of $A$. From Glicksberg's theorem we have $y_{n}=\mathbf{A}(G, A)$ for all $n$ sufficiently large, and hence $x_{n} \in \mathbf{A}(G, A)$ for all $n$ sufficiently large; since $\left\{x_{n}\right\}$ is faithfully indexed it follows that $|\mathbf{A}(G, A)| \geq \omega$. This contradiction gives $\lambda A=0$, as required.

The above result is closely related to $[\mathrm{KN}](7.8)$, and $[\mathrm{Sc}]$.

If $G \in$ LCAG and $N$ is a closed subgroup of bG, then we say that $\phi: G \rightarrow \mathrm{b} G / N$ preserves closed subgroups of $G$ if for any closed subgroup $H$ of $G, \phi[H]$ is closed in $\phi[G]$. For example, if $G$ and $H$ denote the discrete groups $\mathbb{R}_{\mathrm{d}}$ and $\mathbb{Q}_{\mathrm{d}}$ of real and rational numbers respectively, there exists a closed subgroup $N$ of $\mathrm{b} G$ such that $N \cap G^{+}=\{0\}$ and $\mathrm{b} G / N=\mathrm{b} \mathbb{R}$. Since $\phi[H]=\mathbb{Q}^{+}$is not closed in $\phi[G]=\mathbb{R}^{+}, \phi$ does not preserve closed subgroups of $G$.

3.11. Lemma. Let $G$ be a discrete Abelian group and $N$ a closed subgroup of $\mathrm{b} G$ such that $N \cap G=\{1\}$, and let $\lambda$ denote Haar measure of $\widehat{G}$. Suppose that either

(a) $\{\chi \mid G: \chi \in \mathbf{A}(\widehat{\mathrm{b} G}, N)\}$ is not Haar-measurable in $\widehat{G}$, or

(b) $\lambda(\{\chi \mid G: \chi \in \mathbf{A}(\widehat{\mathrm{b} G}, N)\})>0$.

If either $G$ is countable or $\phi: G \rightarrow \mathrm{b} G / N$ preserves closed subgroups of $G$, then $N$ preserves compactness.

P r o of. Suppose instead that there is an infinite subset $A$ of $G$ such that $\phi[A]$ is compact in bG/N. We claim first that in this case there is $C \subseteq G$ such that $|C|=\omega$ and $\phi[C]$ is compact in $\mathrm{b} G / N$. This is obvious in case $G$ is countable: take $C=A$. Assuming $|G|>\omega$, choose $B \subseteq A$ with $|B|=\omega$, 
and set $H=\langle B\rangle \subseteq G$ and $C=H \cap A$. Since $\phi$ preserves closed subgroups of $G$ the group $\phi[H]$ is closed in $\phi[G]$; since $\phi$ is a bijection from $G$ onto $\phi[G]$ we have

$$
\phi[C]=\phi[H \cap A]=\phi[H] \cap \phi[A],
$$

so $\phi[C]$ is compact and the claim is established.

Like every countably infinite, compact space, $\phi[C]$ is second-countable and hence metrizable. Thus there exist a faithfully indexed sequence $x_{n} \in C$, and $p \in C$, such that $\phi\left(x_{n}\right) \rightarrow \phi(p)$ in $\mathrm{b} G / N$. Replacing $x_{n}$ by $x_{n}-p$ if necessary, we assume $\phi\left(x_{n}\right) \rightarrow \phi(1)=N$ in $\mathrm{b} G / N$. Then every $\chi \in$ $\mathbf{A}(\widehat{\mathrm{b} G}, N)$ satisfies $\chi\left(x_{n}\right) \rightarrow \chi(1)=1$, and it follows (defining $A=\{\chi \in \widehat{G}$ : $\left.\left.\chi\left(x_{n}\right) \rightarrow 1\right\}\right)$ that

$$
\{\chi \mid G: \chi \in \mathbf{A}(\widehat{\mathrm{bG}}, N)\} \subseteq A .
$$

Since $A$ is $\lambda$-measurable in $\widehat{G}$ with $\lambda A=0$, we conclude from $(*)$ that $\{\chi \mid G: \chi \in \mathbf{A}(\widehat{\mathrm{bG}}, N)\}$ itself is $\lambda$-measurable and has $\lambda$-measure equal to 0 . This contradiction completes the proof.

3.12. THEOREM. There is a closed, non-metrizable subgroup of $\mathrm{b} \mathbb{Z}$ such that $N \cap \mathbb{Z}=\{0\}$ and $N$ preserves compactness.

Proof. We note first that the usual construction of a Bernstein subset of $\mathbb{R}$ modifies easily to give a non-measurable subgroup $H$ of $\mathbb{T}$ such that $|\mathbb{T} / H|=\mathbf{c}$. (This was pointed out to us by Oscar Masaveu. See [Str] for related and stronger results.) Indeed, let $\left\{F_{\xi}: \xi<\mathbf{c}\right\}$ be an enumeration of all uncountable, closed subsets of $\mathbb{T}$, choose $p_{0}, q_{0} \in F_{0}$ with $q_{0} \notin\left\langle p_{0}\right\rangle$ and recursively, if $\xi<\mathbf{c}$ and $p_{\eta}, q_{\eta}$ have been chosen for all $\eta<\xi$, choose $p_{\xi}, q_{\xi} \in F_{\xi}$ with

$$
p_{\xi} \notin\left\langle\left\{p_{\eta}: \eta<\xi\right\} \cup\left\{q_{\eta}: \eta<\xi\right\}\right\rangle, \quad q_{\xi} \notin\left\langle\left\{p_{\eta}: \eta \leq \xi\right\} \cup\left\{q_{\eta}: \eta<\xi\right\}\right\rangle .
$$

(The availability of such $p_{\xi}, q_{\xi}$ derives from the fact that $\left|F_{\xi}\right|=\mathbf{c}$.) The group $H=\left\langle\left\{p_{\xi}: \xi<\mathbf{c}\right\}\right\rangle$ is as required: (a) $|\mathbb{T} / H|=\mathbf{c}$, since $\eta<\xi<\mathbf{c}$ yields $q_{\eta}+H \neq q_{\xi}+H$; (b) $\lambda H>0$ is impossible, since that inequality implies that $H$ is open in $\mathbb{T}$ (by the Steinhaus-Weil theorem) and then $H=\mathbb{T}$; and (c) $\lambda H=0$ is impossible, since if $\mathbb{T} \backslash H$ is measurable and $\lambda(\mathbb{T} \backslash H)>0$ then there is a (necessarily uncountable) compact subset $F=F_{\xi}$ of $\mathbb{T} \backslash H$ with $\lambda F>0$, so that $p_{\xi} \in F \subseteq(\mathbb{T} \backslash H) \cap H=\emptyset$.

Now set $N=\mathbf{A}(\mathrm{b} \mathbb{Z}, H)$. Then:

(a) $N$ is a closed subgroup of $\mathrm{b} \mathbb{Z}$;

(b) $N$ is not metrizable, since a compact metrizable space has countable weight while

$$
\mathrm{w} N=|\widehat{N}|=|\widehat{\mathrm{b}} / H|=|\mathbb{T} / H|=\mathbf{c} ;
$$

(c) $N \cap \mathbb{Z}=\{0\}$ since $H$ is dense in $\mathbb{T}$; and 
(d) $\{\chi \mid \mathbb{Z}: \chi \in \mathbf{A}(\widehat{\mathrm{b}}, N)\}=H$ is not measurable in $\widehat{\mathbb{Z}}=\mathbb{T}$, so $N$ preserves compactness by Lemma 3.11.

4. Some questions. The results proved above raise some questions which our methods seem inadequate to settle. Perhaps the most interesting of these is 4.1 , which was contributed by the referee.

We hope to return to some of these in a later communication.

4.1. Question. Let $G \in$ MAP and suppose that every closed, metrizable subgroup $N$ of b $G$ such that $N \cap G=\{1\}$ preserves compactness (in the sense of Definition 2.1(a)). Does it follow that $G$ strongly respects compactness?

Of course, Theorem 2.10 responds affirmatively to Question 4.1 (even if the hypothesis $N \cap G=\{1\}$ is omitted) if the assumption $G \in \mathbf{M A P}$ is strengthened to $G \in \mathbf{L C A G}$.

4.2. Question. Characterize those MAP groups which strongly respect compactness.

4.3. Question. Characterize those $G \in$ TBAG such that $\mathrm{k} G \in \mathbf{L C A G}$ and $(\mathrm{k} G)^{+}=G$.

4.4. Question. Given $G \in \mathbf{L C A G}$ and a closed subgroup $N$ of bG such that $N \cap G=\{0\}$, clarify the relation between the properties " $N$ preserves compactness" and " $\phi: G \rightarrow \mathrm{b} G / N$ preserves closed subgroups of $G$."

4.5. Question. Suppose that in Lemma 3.11 the hypothesis " $G$ is countable or $\phi$ preserves closed subgroups" is deleted. Is the resulting statement valid?

4.6. Question. Let $G=\langle G, \mathcal{T}\rangle \in$ LCAG and let $N$ be a closed subgroup of bG such that $N \cap G$ is metrizable. Suppose that every subset $A$ of $G$ satisfies: $A \cdot(N \cap G)$ is compact in $G$ if and only if $\phi[A]$ is compact in b $G / N$. What can be said about $N$ ?

\section{References}

[C] W. W. Comfort, Topological groups, in: Handbook of Set-Theoretic Topology, K. Kunen and J. Vaughan (eds.), North-Holland, Amsterdam, 1984, 1143-1263.

[CHR] W. W. Comfort, K.-H. Hofmann and D. Remus, Topological groups and semigroups, in: Recent Progress in General Topology, M. Hušek and J. van Mill (eds.), North-Holland, Amsterdam, 1992, 57-144.

[CR] W. W. Comfort and K. A. Ross, Topologies induced by groups of characters, Fund. Math. 55 (1964), 283-291.

[CT] W. W. Comfort and F. J. Trigos-Arrieta, Remarks on a Theorem of Glicksberg, in: General Topology and Applications, S. J. Andima, R. Kopperman, 
P. R. Misra, J. Z. Reichman and A. R. Todd (eds.), Lecture Notes in Pure and Appl. Math. 134, Dekker, New York, 1991, 25-33.

[DPS] D. N. Dikranjan, I. R. Prodanov and L. N. Stoyanov, Topological Groups, Lecture Notes in Pure and Appl. Math. 130, Dekker, New York, 1989.

[E] R. Engelking, General Topology, Monografie Mat. 60, PWN-Polish Scientific Publishers, Warszawa, 1977.

[F] P. Flor, Zur Bohr-Konvergenz von Folgen, Math. Scand. 23 (1968), 169-170.

[G] I. Glicksberg, Uniform boundedness for groups, Canad. J. Math. 14 (1962), 269-276.

[HR] E. Hewitt and K. A. Ross, Abstract Harmonic Analysis, Vol. I, Grundlehren Math. Wiss. 115, Springer, Berlin, 1963.

[Hu] R. Hughes, Compactness in locally compact groups, Bull. Amer. Math. Soc. 79 (1973), 122-123.

[KN] L. Kuipers and H. Niederreiter, Uniform Distribution of Sequences, Wiley, New York, 1974

[K] V. Kuz'minov, On a hypothesis of P. S. Alexandrov in the theory of topological groups, Dokl. Akad. Nauk SSSR 125 (1959), 727-729 (in Russian).

[L] W. F. LaMartin, On the foundations of k-group theory, Dissertationes Math. 146 (1977).

[Mo] W. Moran, On almost periodic compactifications of locally compact groups, J. London Math. Soc. (2) 3 (1971), 507-512.

[Re] G. A. Reid, On sequential convergence in groups, Math. Z. 102 (1967), 227-235.

[RT] D. Remus and F. J. Trigos-Arrieta, Abelian groups which satisfy Pontryagin duality need not respect compactness, Proc. Amer. Math. Soc. 117 (1993), $1195-1200$.

[Ro] K. A. Ross, Commentary on selected papers of S. Kakutani, in: S. Kakutani, Selected Papers, Vol. I, Robert A. Kallman (ed.), Birkhäuser, Boston, 1986.

$[\check{S}] \quad$ B. È. Šapirovskiǔ, On embedding extremally disconnected spaces in compact Hausdorff spaces. b-points and weight of pointwise normal spaces, Dokl. Akad. Nauk SSSR 223 (1975), 1083-1086 (in Russian); English transl.: Soviet Math. Dokl. 16 (1975), 1056-1061.

[Sc] I. J. Schoenberg, Asymptotic distribution of sequences (solution of Problem 5090), Amer. Math. Monthly 71 (1964), 332-334.

[Sh] D. B. Shakhmatov, A direct proof that every infinite compact group $G$ contains $\{0,1\}^{w(G)}$, in: Papers in General Topology and Applications, Proc. June 1992 Queens College Conference, Ann. New York Acad. Sci., New York, to appear.

[Sm] Yu. M. Smirnov, On the metrizability of bicompacta decomposable into a union of sets with countable basis, Fund. Math. 43 (1956), 387-393 (in Russian).

[Sto] A. H. Stone, Metrisability of unions, Proc. Amer. Math. Soc. 10 (1959), 361-366.

[Str] K. R. Stromberg, Universally nonmeasurable subgroups of $\mathbb{R}$, Amer. Math. Monthly 99 (1992), 253-255.

[T1] F. J. Trigos-Arrieta, Pseudocompactness on groups, doctoral dissertation, Wesleyan University, 1991.

[T2] —, Pseudocompactness on groups, in: General Topology and Applications, Lecture Notes in Pure and Appl. Math. 134, S. Andima et al. (eds.), Dekker, New York, 1991, 369-378.

[T3] - Continuity, boundedness, connectedness and the Lindelöf property for topological groups, J. Pure Appl. Algebra 70 (1991), 199-210 (= Proceedings of the 1989 Curaçao Conference on Locales and Topological Groups). 
[Ve] R. Venkataraman, Compactness in Abelian topological groups, Pacific J. Math. 57 (1975), 591-595.

[Vi] N. Ya. Vilenkin, On the dyadicity of the group space of bicompact commutative groups, Uspekhi Mat. Nauk 13 (6) (84) (1958), 79-80 (in Russian).

[W] A. Weil, Sur les espaces à structure uniforme et sur la topologie générale, Publ. Math. Univ. Strasbourg, Hermann, Paris, 1937.

DEPARTMENT OF MATHEMATICS

WESLEYAN UNIVERSITY

MIDDLETOWN, CONNECTICUT 06459

U.S.A.

E-mail:WCOMFORT@EAGLE.WESLEYAN.EDU

DEPARTMENT OF MATHEMATICS

CALIFORNIA STATE UNIVERSITY

BAKERSFIELD, CALIFORNIA 93311-1099

U.S.A.

E-mail: JTRIGOS@CSBINA.CSUBAK.EDU
DEPARTMENT OF MATHEMATICS CASE WESTERN RESERVE UNIVERSITY CLEVELAND, OHIO 44106-7058 U.S.A.

Received 7 April 1992;

in revised form 2 December 1992 and 14 May 1993 